Article

\title{
The Unexpected Fractal Signatures in Fibonacci Chains
}

\author{
Fang Fang $\mathbb{D}$ *, Raymond Aschheim $(\mathbb{D}$ and Klee Irwin $\mathbb{D}$
}

Quantum Gravity Research, Los Angeles, CA 90290, USA; raymond@quantumgravityresearch.org (R.A.);

Klee@quantumgravityresearch.org (K.I.)

* Correspondence: Fang@quantumgravityresearch.org

Received: 2 August 2019; Accepted: 29 October 2019; Published: 6 November 2019

Abstract: In this paper, a new fractal signature possessing the cardioid shape in the Mandelbrot set is presented in the Fourier space of a Fibonacci chain with two lengths, $L$ and $S$, where $L / S=\phi$. The corresponding pointwise dimension is 1.7. Various modifications, such as truncation from the head or tail, scrambling the orders of the sequence and changing the ratio of the $L$ and $S$, are done on the Fibonacci chain. The resulting patterns in the Fourier space show that that the fractal signature is very sensitive to changes in the Fibonacci order but not to the $L / S$ ratio.

Keywords: Fibonacci chain; fractal signature; Fourier space

\section{Introduction}

Quasicrystals possess exotic and sometimes anomalous properties that have interested the scientific community since their discovery by Shechtman in 1982 [1]. Of particular interest in this manuscript is the self similar property of quasicrystals that links them to fractal. Historically, research on the fractal aspect of quasicrystalline properties has revolved around spectral and wave function analysis [2-5]. Mathematical investigation [6-9] of the geometric structure of quasicrystals is less represented in the literature than experimental work.

In this paper, a new framework for analyzing the fractal nature of quasicrystals is introduced. Specifically, the fractal properties of a one-dimensional Fibonacci chain and its variations are studied in the complex Fourier space. The results may also be found in two and three dimensional quasicrystals that can be constructed using a network of one dimensional Fibonacci chains [10].

\section{The Fractal Signature of the Fibonacci Chain in Fourier Space}

The Fibonacci chain is a quasiperiodic sequence of short and long segments where the ratio between the long and the short segment is the golden ratio [11]. It is an important 1D quasicrystal that uniquely removes the arbitrary closeness in the non-quasicrystalline grid space of a quasicrystal and as a result the non-quasicrystalline grid space is converted into a quasicrystal as well [10]. The Fourier representation of a Fibonacci chain is given as follows:

$$
z_{s}=\frac{1}{\sqrt{n}} \sum_{r=1}^{n} u_{r} e^{\frac{2 \pi i(r-1)(s-1)}{n}}, s=1,2, \ldots n,
$$

where

$$
u_{r}=(\phi-1)(\lfloor(r+1) \phi\rfloor-\lfloor r \phi\rfloor-2)+\phi=(\phi-1)(\lfloor(r+1) \phi\rfloor-\lfloor r \phi\rfloor)+(2-\phi), r=1,2, \ldots n
$$

is a Fibonacci chain of length $n$ with units of length $\phi$ and 1, where $x \rightarrow\lfloor x\rfloor$ is the floor function. Here $\phi=\frac{\sqrt{5}+1}{2}$ is the golden ratio. 
Note that the Fibonacci chain can also be generated using substitution rules $\{L \rightarrow L S, S \rightarrow L\}$ [11] where $m-1$ iterations of the substitution method produces a Fibonacci chain of length (its number of tiles) equal to the $m$ th Fibonacci number $F_{m}:|S|=1=F_{1},|L|=1=F_{2},|L S|=2=F_{3},|L S L|=3=$ $F_{4},|L S L L S|=5=F_{5}, \ldots$. Tiles $L$ and $S$ (for Long and Short) are of respective length $\phi$ and 1 and $u_{r}$ given by Equation (2) is the coordinate of the right point of the $r$ th tile.

Consecutive iterations of the Fibonacci chain are plotted in the Fourier space in Figure 1. Figure 2 shows that the main cardioid (heart shape) in the Mandelbrot set [12] appeared at the center of each plot. It is known that the denominators of the periods of the circular bulbs at sequential scales in the Mandelbrot set follow the Fibonacci number sequence [13].

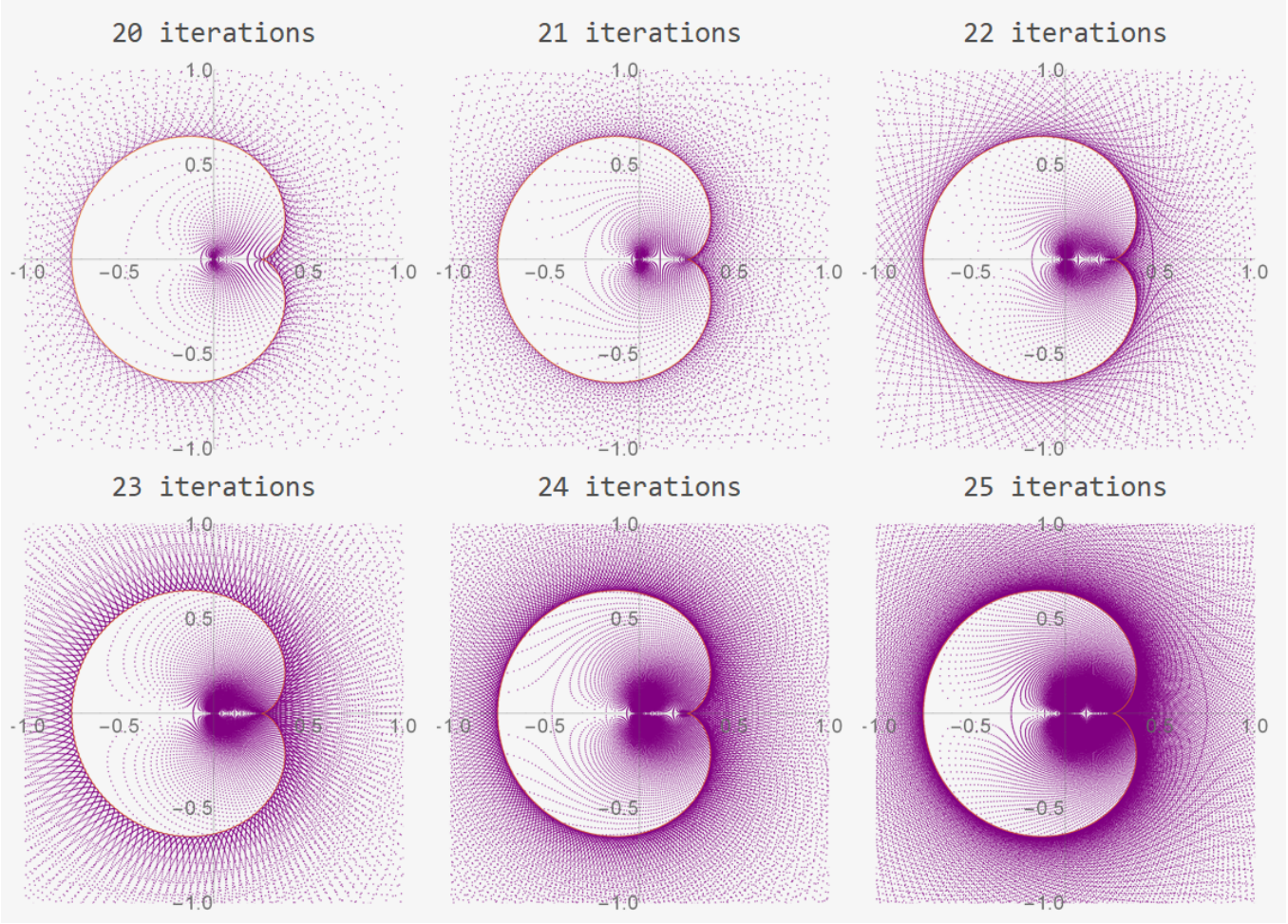

Figure 1. The rescaled Fourier space representation of a Fibonacci chain is shown here using the substitution method with different iterations, starting with 20 . The horizontal axis represents the real part and the vertical axis represents the imaginary part of the Fourier coefficients.

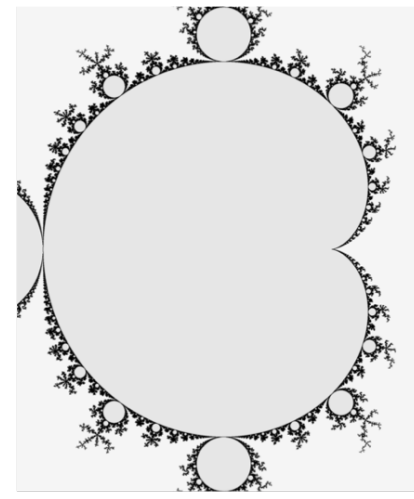

a

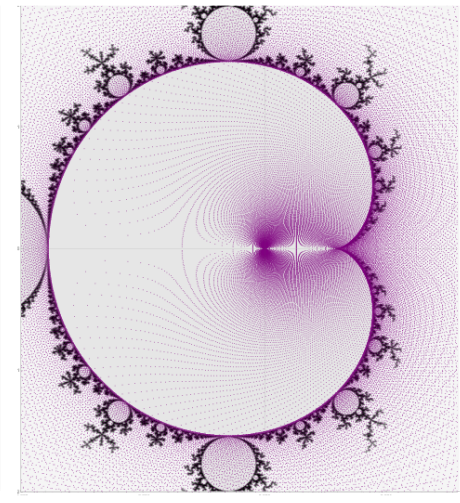

b

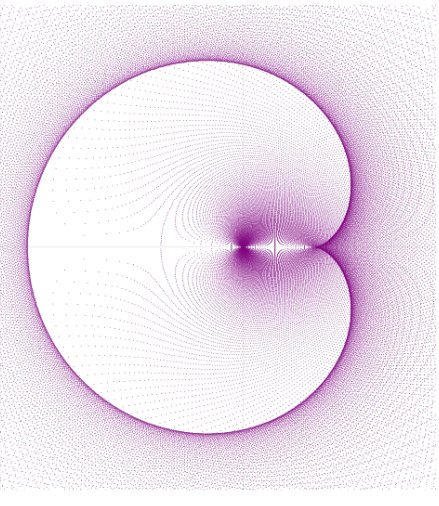

C

Figure 2. (a) The Mandelbrot set, (b) overlays of the Mandelbrot set and the Fourier space with matching cardioid, and (c) the fractal structure in the Fourier space of a Fibonacci chain with 25 iterations. 
The plots reveal that the size of the cardioid decreases with successive iterations and the scaling factor for the size change is approximately $1 / \sqrt{\phi}$ and precisely $\sqrt{\frac{F_{m-1}}{F_{m}}}$, so we can rescale the Equation (1) by omitting the normalisation factor $\sqrt{\frac{1}{F_{m}}}$.

$$
z_{s, m}=\sum_{r=1}^{F_{m}} u_{r} e^{\frac{2 \pi i(r-1)(s-1)}{F_{m}}}, s=1,2, \ldots F_{m}
$$

Section 2.3 will show that each momentum (point in the complex plane of the Fourier transform) $z_{s, m}$ is very close to a point $z_{p, m+4}$ in the point set obtained with four more iterations. Conveniently rearranging the terms from Equation (3) gives:

$$
z_{1+F_{k}, m} \approx(-\phi)^{2 k-1-m}
$$

Due to the minus sign before $\phi$, the orientation of the cardioid undergoes mirror flips with every odd and even numbered iteration. Upon magnification, the fractal nature becomes apparent near the real axis, as shown in Figure 3.

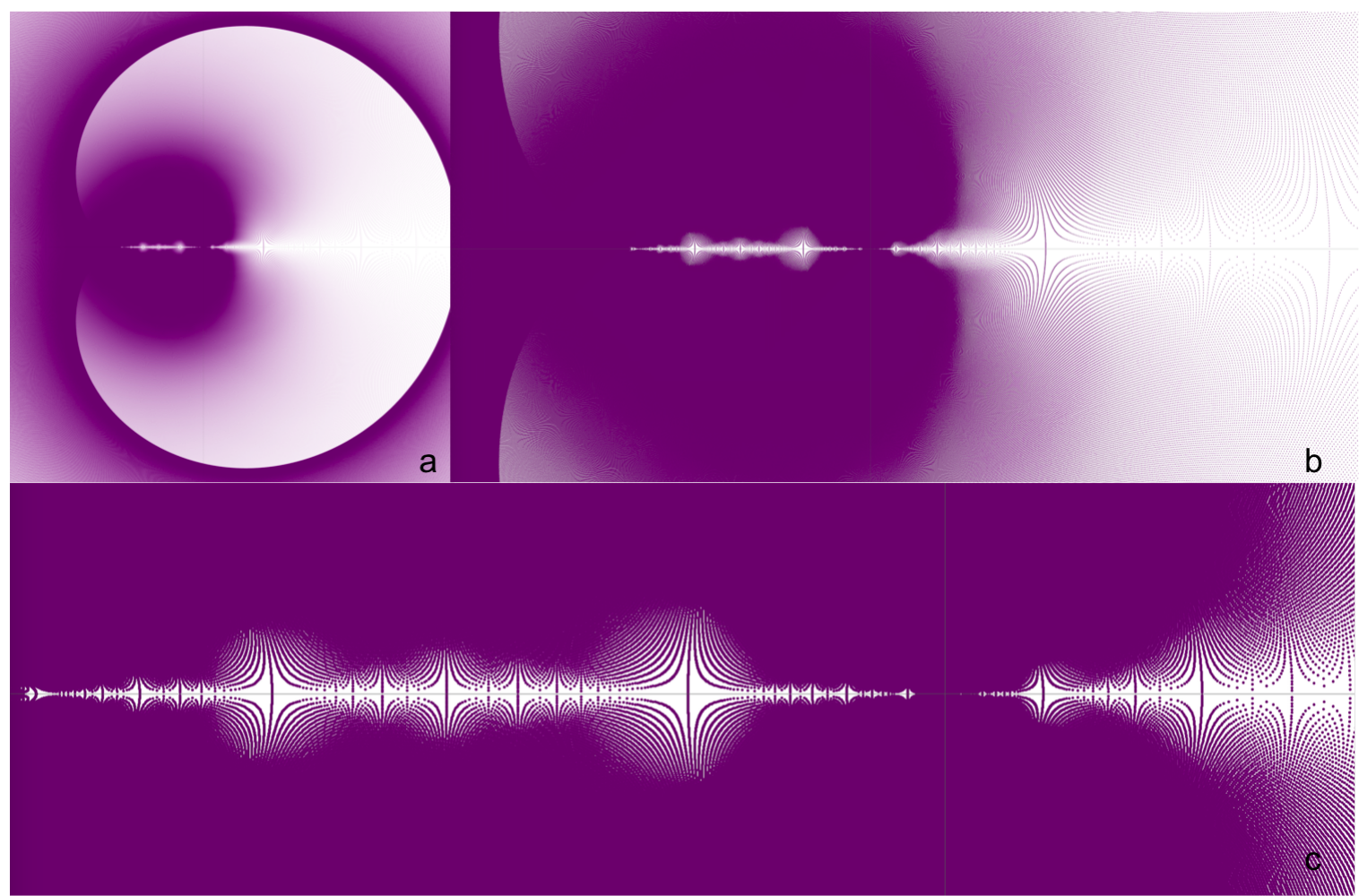

Figure 3. Appearance of the fractal pattern at the 34th iteration of the Fibonacci chain: (a) general view showing the cardioid; (b) detail of the central part; (c) zoom near the real axis, some lines are getting very close and perpendicular to the real axis, they are called 'trunks' in Figure 6.

\subsection{Fractal Dimension}

The fractal dimension of the complex Fourier representation of the Fibonacci chain is calculated as the pointwise dimension proposed by Grassberger and Procaccia [14]. The system we are studying here is a chaotic system that settles down to a strange attractor in phase space. Fixing a point $x$ on the attractor $\mathrm{A}$, the number of points, $N_{x}(\epsilon)$, on $\mathrm{A}$ inside a ball of radius $\epsilon$ about $x$, typically grows as a power law: $N_{x}(\epsilon) \propto \epsilon^{d}$, where $d$ is called the pointwise dimension. It is calculated to be approximately 1.7 for the complex Fourier representation of the Fibonacci chain (Figure 4). 


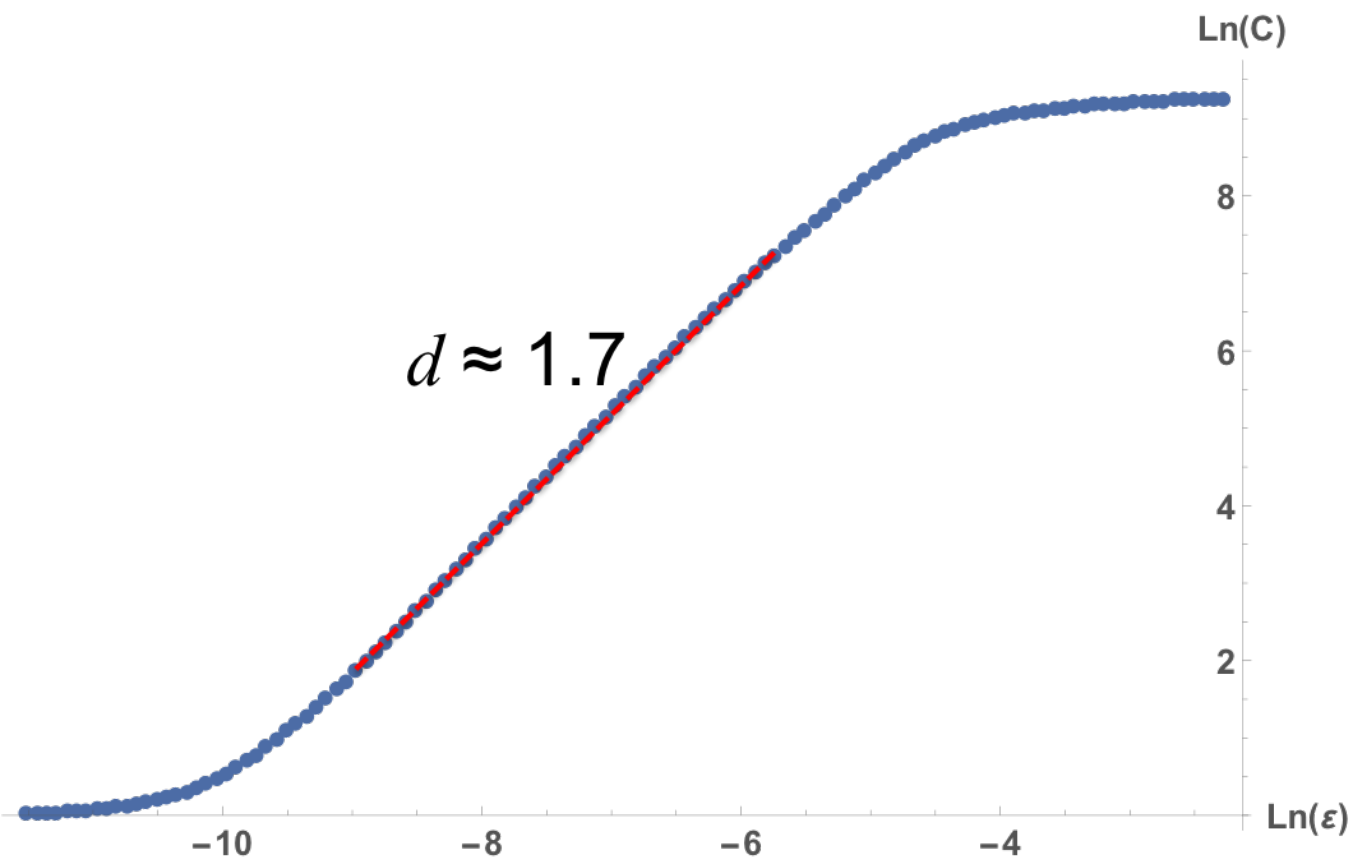

Figure 4. Pointwise dimension of the of the Fibonacci chain in Fourier space.

\subsection{Universality Near the Real Line}

The well-known Mandelbrot set is a collection of complex numbers $c$ satisfying the equation $z_{n+1}=z_{n}^{2}-c$ where $z_{n+1}<2$. With each fixed point $c$ there is a Julia set. The universality emerging near the real line is related to the non-fractal limit of the Julia set, at the specific point $c=-2$, which corresponds tp a fixed point for $z_{0}=-2$. There is no more Julia set at this point, like at the trivial fixed point $c=0$. But there is a very chaoticly looking fractal concentrated around the real line when $c=-2+\epsilon$. When sliding $c$ along the real line from $c=-1$ to $c=-2$, a set of attractors and anti-attractors are also sliding and converging to a limit (Figure 5).

The sequence $z_{s}$ seems to be sparse and not organized locally, as the neighbours are not local but as in every quasicrystal there is a non local order. Near the real line are seeded trees of a non planar grid which self-organize in modular geodesics of lengths of the Fibonacci numbers, which are measured as the difference of indices.

$$
\mathcal{C}_{m}^{v}=z_{1+v F_{m}} ; \quad \mathcal{C}_{m}^{v,+h}=z_{1+v F_{m}+h F_{m+2}} ; \quad \mathcal{C}_{m}^{v,-h}=z_{1+v F_{m}+h F_{m-2}}
$$

$\mathcal{C}_{m}^{v}$ is a seed for any $m$ and $\mathrm{v}=1$ and it equals to the complex number $z_{s, m}$ defined by Equation (3) where $s=6,8,14,22,56,90 \ldots$ close to the real line because of Equation (4). For $\mathcal{C}_{m}^{1}(k)=z_{1+F_{k}, m}$, a vertical trunk emerges from this seed perpendicular to the real line and realizes a modular geodesic of $z_{s, m}$ where the index $s$ grows by a multiple of $F_{m}$. Therefore from the seed $z_{56}$ grows the trunk $z_{111}, z_{166}, z_{221}, z_{276}$ and from the seed $z_{35}$ grows the trunk $z_{69}, z_{103}, z_{137}, z_{171}$ (Figure 6).

\subsection{Self Similarity}

Equation (4) enables a map from $\mathbb{C}$ to itself, mapping the iteration $m$ to a subset of the iteration $m+4$. It maps seeds of different periodicity and also their corresponding trees. This proves that the iteration $m+4$ contains a scaled version of the iteration $m$ and that the sequence of spectrum is self-similar and becomes fractal at the infinite limit $m \rightarrow \infty$. 

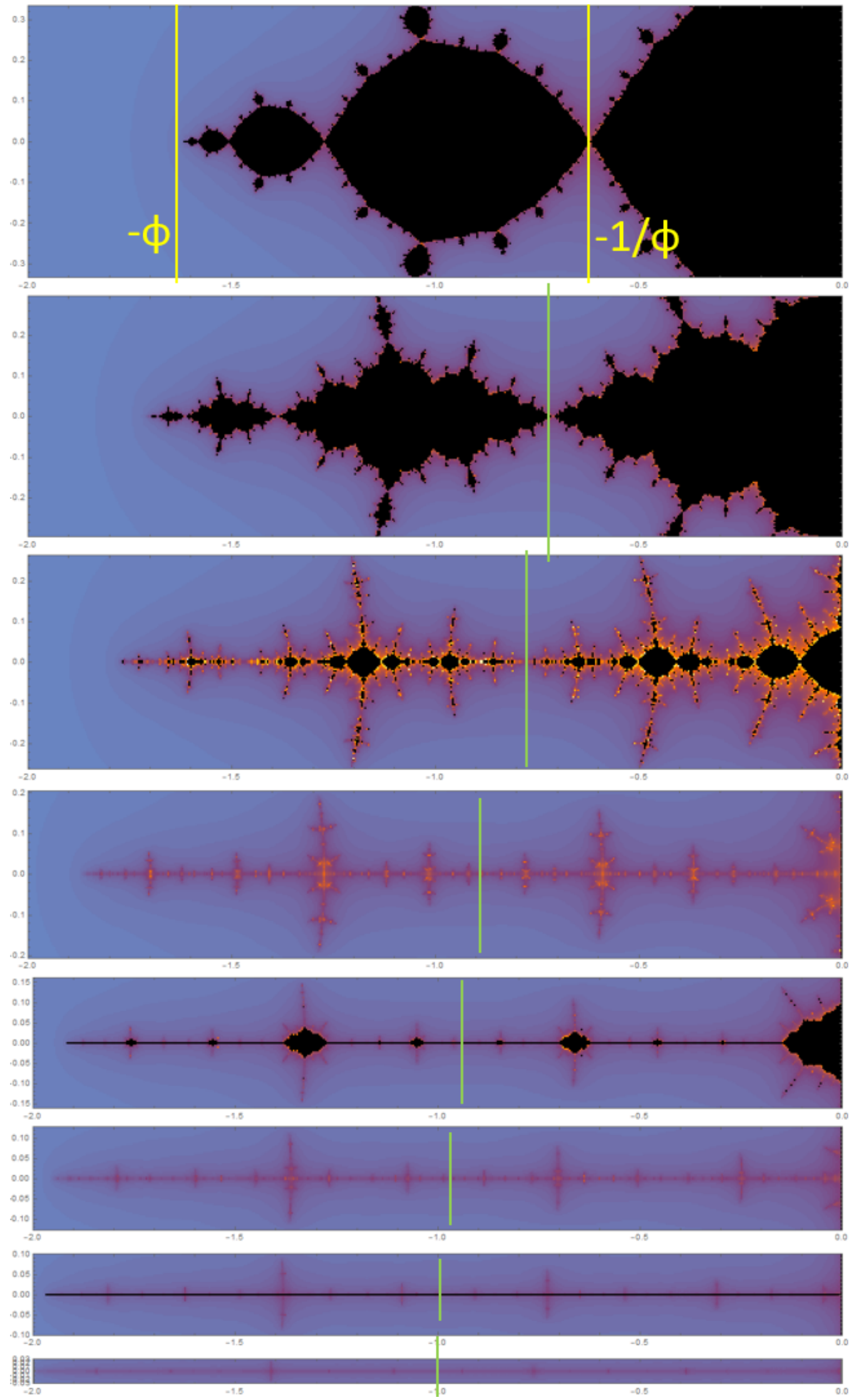

Figure 5. The Julia sets on the real line, while sliding from the Golden julia set (on the top) where $c=-1$ to the limit of non fractality, $c=-2$ on the bottom, shows a sliding of the attractors and anti-attractors. For example the left end slides from $-\phi$ to -2 . 


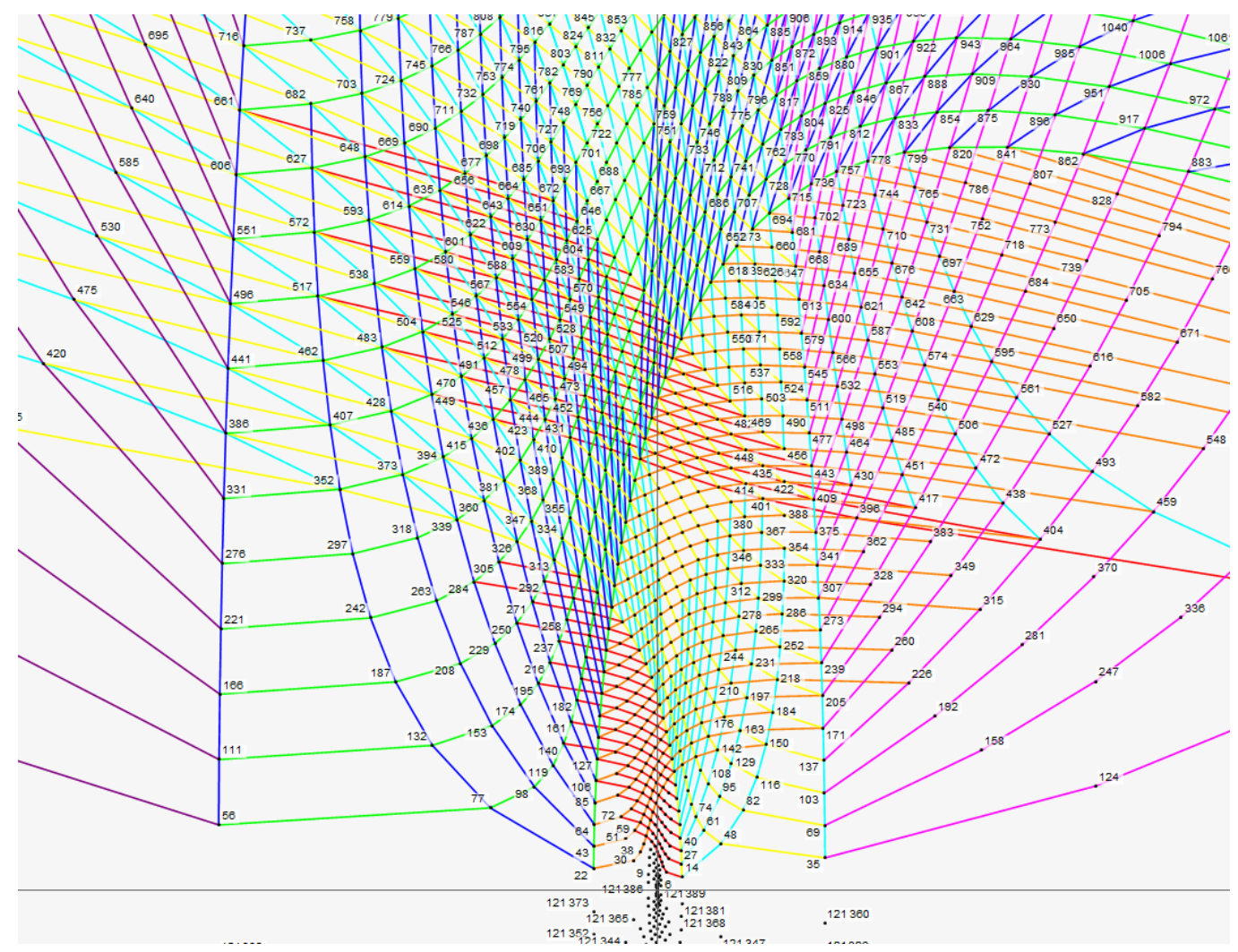

Figure 6. The Fourier space representation of a Fibonacci chain is shown here with the geodesics of Fibonacci steps: 5 in red, 8 in orange, 13 in yellow, 21 in green, 34 in cyan, 55 in blue, 89 in magenta and 144 in purple. The "seeds" are the point closest to the horizontal axis: 56 on the left, 22 spreading a green trunk, 14 with a yellow trunk and 35 with a cyan trunk. The other trees are omitted.

\section{The Variations of the Fibonacci Chain in Fourier Space}

It is tempting to conjecture that this kind of Fractal signature will appear in any finite portion of the Fibonacci chain or a Fibonacci chain that is nearly perfect (a Fibonacci chain that is generated strictly from the substitution rules mentioned earlier). A series of tests has been conducted to verify this conjecture. The results are surprising. Figure 7 shows the results of the Fourier space of the Fibonacci chain of 25th iteration with various, mostly minor modifications, such as, (Figure 7a-f) small truncation from the head or tail of the chain, (Figure 71-p) scrambling the order of a very small part of the chain or (Figure $7 \mathrm{~s}$ ) changing of the $L / S$ ratio. These results show that the fractal pattern, especially the cardioid shape, is very sensitive to any modification except changing the $L / S$ ratio, in which case only the scaling of the fractal pattern changes. A linear transformation of the size of the two tiles $S_{1} \rightarrow S_{2}$ and $L_{1} \rightarrow L_{2}$ expressed as $u_{r}^{\prime}=f\left(u_{r}\right)=a u_{r}+b$ provides by linearity of the Fourier transform the scaled result as observed. In other words, the fractal pattern is a direct result of the substitution rule (or inflation/deflation rule) which gives the Fibonacci ordering of the $L$ and $S$. The exact value of the $L$ and $S$ does not matter for the pattern. Even the smallest breakdown of the rule will result in a non-prefect-closure of the cardioid and therefore result in a completely different pattern. It is similar to the butterfly effect in chaos theory.

\subsection{Variations by Cyclic Permutations}

A cyclically permuted Fibonacci chain $u_{r_{p}}$ where $r_{p}=\operatorname{Mod}\left[r+\left\lfloor\frac{n}{p}\right\rfloor, n\right]$, is created, based on Equation (2). For example when $n=F_{m}=F_{18}$ we get 100 possible value of $p$ and corresponding permutations of $r_{p}$ when $\left\lfloor\frac{n}{p}\right\rfloor \in\{1,2,3, \ldots 646,861,1292,2584\}$. Superposing these 100 Fourier spectra in one image reveals the formation of rings of various density, separated by rings of zero density, 
as in Figure 7q. This shows that the cyclic permutation results in the changing of the phases in the Fourier spectrum.

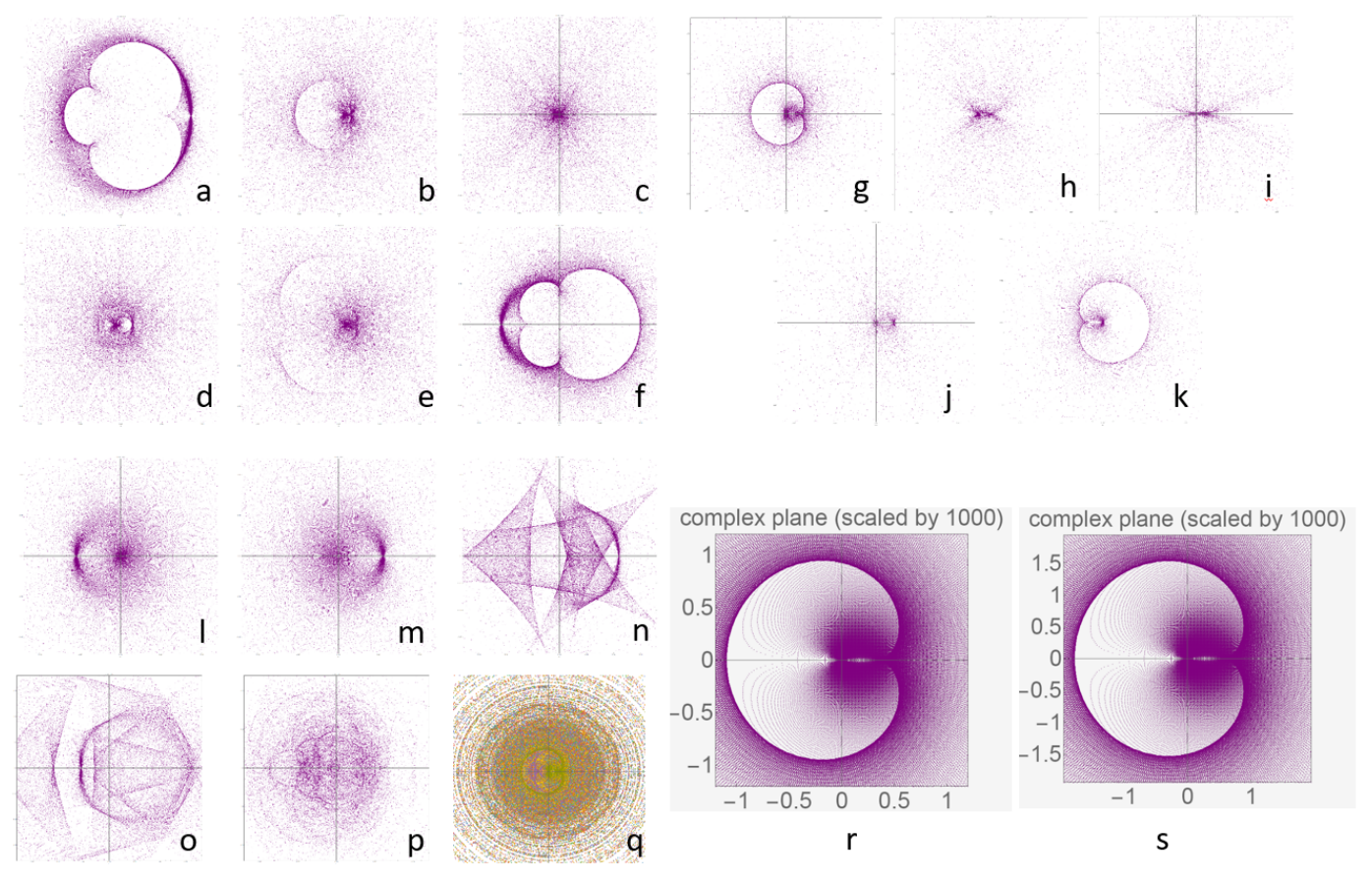

Figure 7. The Fourier space representation of a Fibonacci chain of 25th iteration, with (a) the first segment removed; (b) the first two segments removed; (c) the first three segments removed; (d) the first six segments removed, (e) the first seven segments removed, (f) the first segment length replaced with 0; (g) no modification; (h) the last segment removed, (i) the last two segments remove; (j) the last 46,367 segments removed; (k) the last 46,368 segments removed and the Fibonacci chain of 25th iteration is truncated to the Fibonacci chain of 24th iteration; (1) the 1st segment $L$ replaced with $S$; (m) the last two segments flipped order; (n) the order of the last five segments scrambled; (o) the order of the last ten segments scrambled; (p) the order of the last 100 segments scrambled, (q) the superposition of spectra of cyclic permutations of the 17th iteration and the comparison between (r) the original Fibonacci chain of 27 th iteration and (s) the chain with modified $L / S$ ratio where $L / S=2$.

\subsection{Variations and the Generalized Mandelbrot Set}

Some variations reminds one the Mandelbrot sets of higher degree, named generalized Mandelbrot set.

$$
z_{n+1}=z_{n}^{p}+c
$$

The exponent $p$ equals to 2 for the Mandelbrot set and will give a cardioid (Figure $7 \mathrm{~g}$ ).

$$
z(t)=x+i y=\frac{e^{i t}}{2}-\frac{e^{2 i t}}{4}
$$

The power $p$ can be 3 and give a Mandelbrot set-like fractal with two bulbs and the cardioid will be replaced by a nephroid (like Figure 7e). And when $p=4$ there will be 3 bulbs, (Figure 7a); when $p=5$ there will be 4 bulbs (Figure 7f). The symmetries observed in the derivative Fourier spectrum are the same as the main feature of the generalised Mandelbrot set illustrated in Figure 8 . The mathematical relationship is a result of the fact that both these curves and the Fourier spectrum are sum of imaginary exponentials. There is a subset of the discrete Fourier spectrum set which are very close to the points from Equation (4) after rescaling. It is more hidden but similar for the higher order.

Its worth noting that derivative patterns resemble the cycloidal pattern drawn by a geometric chuck. Figure 9 shows pictures taken from a book published 100 years ago by Bazley [15]. Figure 9a is 
a picture of a geometric chuck. Figure $9 b-d$ are a few cycloids created by the geometric chuck with different settings on the chuck. The fractal signature of a perfect Fibonacci chain may correspond to some perfect quasiperiodic gearing between the plates of the geometric chuck.

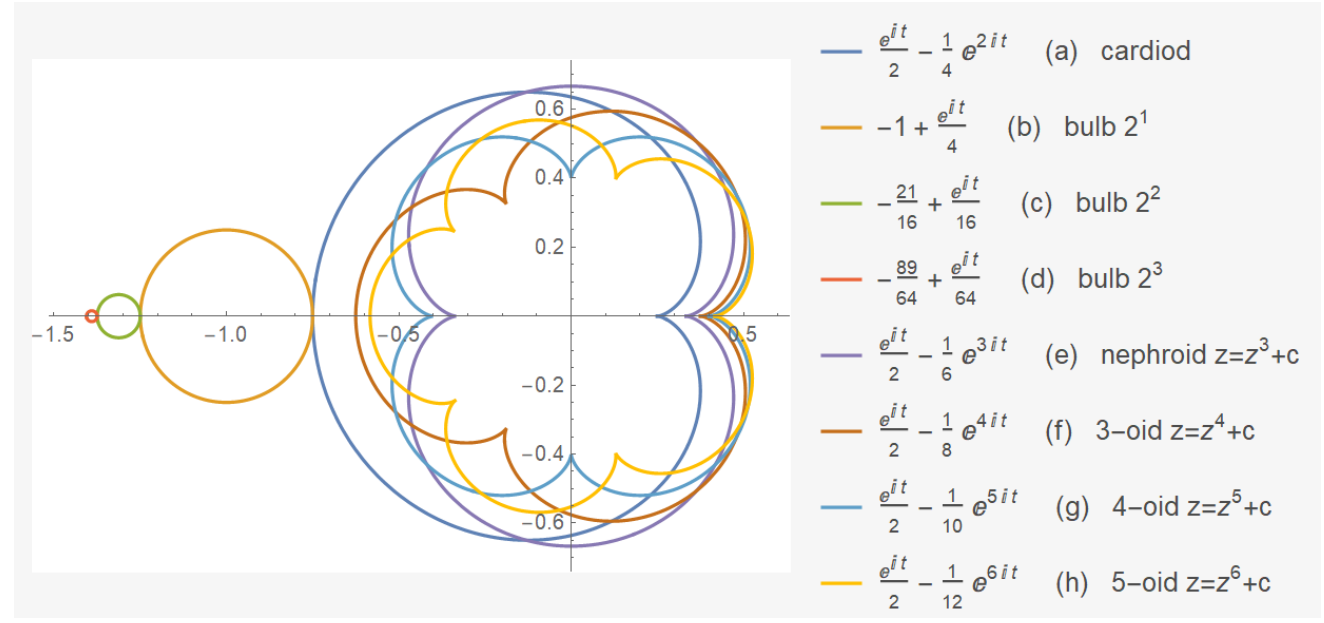

Figure 8. Curves on the complex plane, from main features of the generalised Mandelbrot sets, with their equations; (a-d) Epicycloid from the Mandelbrot set and its 3 first circular bulbs centered on the real line (e-h) Nephroid and its higher generalisations from the generalized Mandelbrot set for exponents 3 to 6 .
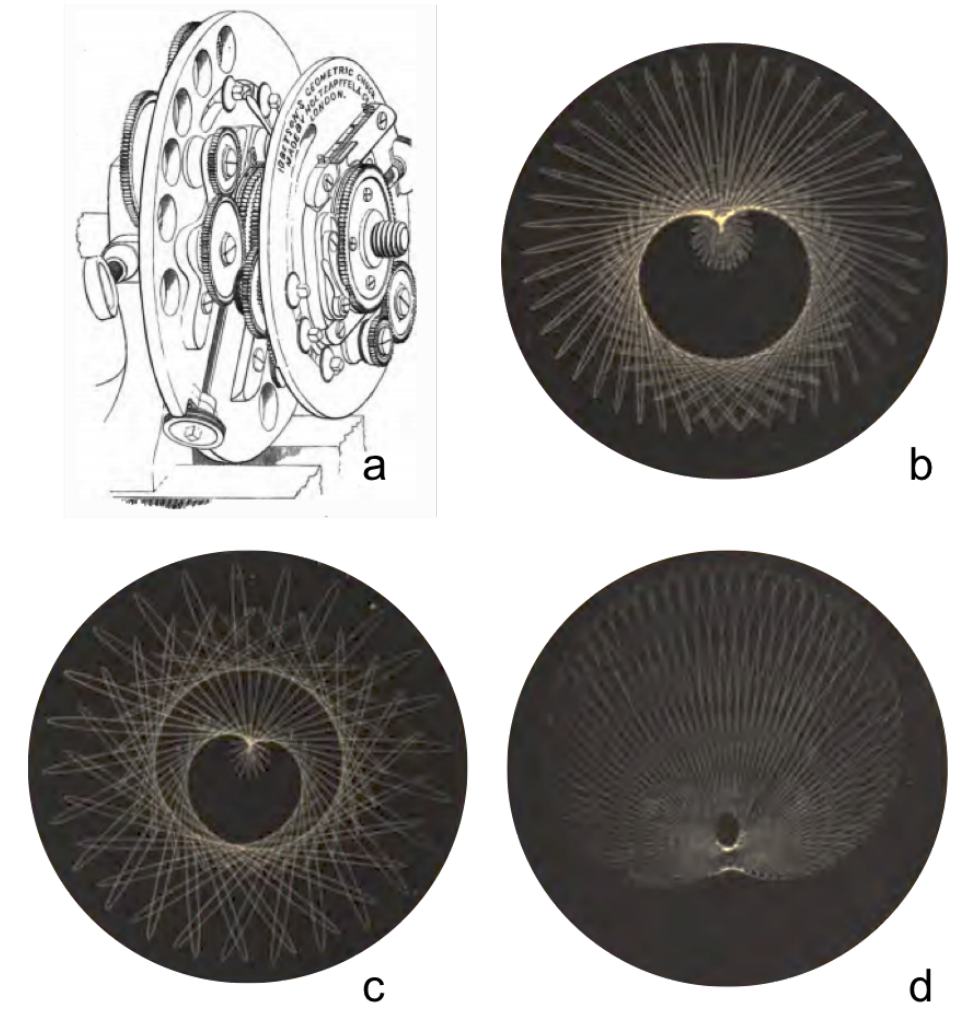

Figure 9. (a) A picture of a geometric chuck; (b-d) Cycloids generated by the geometric chuck with different settings. The following settings are used: $P=55, V_{1}=\frac{111}{55}=\frac{2 P+1}{P},(\mathbf{a}): V_{2}=55 \mathrm{out}, E x_{1}=30$, $E x_{2}=55, S R=27,(\mathbf{b}): V_{2}=55 \mathrm{in}, E x_{1}=35, E x_{2}=45, S R=30,(\mathbf{c}): V_{2}=\frac{55}{2}$ out $, E x_{1}=30, E x_{2}=55$, $S R=27$, The equation is not given but the information in Reference [15] should help with finding it. 


\section{Summary}

This paper reports a novel fractal analysis of a Fibonacci chain in Fourier space by computing the pointwise dimensions and visual inspection of the Fourier pattern. The cardioid structure in the Mandelbrot set is observed for the case of the one dimensional Fibonacci chain, with alternating mirror symmetry according to odd or even instances of the substitution iteration that generates the Fibonacci chains. The Fourier pattern is very sensitive to how strictly the chain follows the substitution rules. Any small variation in that will completely change the pattern. In comparison, the change in the $L / S$ ratio has no influence in the Fourier pattern other than a change in scaling.

Author Contributions: Conceptualization, F.F. and K.I.; Investigation, F.F., R.A. and K.I.; Methodology and Writing Manuscript, F.F. and R.A.; Software, F.F. and R.A.; Supervision, K.I.

Funding: This research received no external funding.

Conflicts of Interest: The authors declare no conflict of interest.

\section{References}

1. Shechtman, D.; Blech, I.; Gratias, D.; Cahn, J.W. Metallic phase with long-range orientational order and no translational symmetry. Phys. Rev. Lett. 1984, 53, 1951. [CrossRef]

2. Strichartz, R.S. Differential Equations on Fractals; Princeton University Press: Princeton, NJ, USA, 2006.

3. Grimm, U.; Schreiber, M. Energy spectra and eigenstates of quasiperiodic tight-binding hamiltonians. arXiv 2002, arXiv:cond-mat/0212140.

4. Repetowicz, P.; Grimm, U.; Schreiber, M. Exact eigenstates of tight-binding hamiltonians on the penrose tiling. arXiv 1998, arXiv:cond-mat/9805321.

5. Macé, N.; Jagannathan, A.; Piéchon, F. Fractal dimensions of the wavefunctions and local spectral measures on the fibonacci chain. arXiv 2016, arXiv:1601.00532.

6. Luck, J.M.; Godreche, C.; Janner, A.; Janssen, T. The nature of the atomic surfaces of quasiperiodic self-similar structures. J. Phys. A 1993, 26, 1951. [CrossRef]

7. Baake, M.; Grimm, U. Aperiodic Order. In Encyclopedia of Mathematics and its Applications; Vol. 1: A Mathematical Invitation; Cambraidge University Press: Cambraidge, UK, 2013.

8. Ramachandrarao, P.; Sina, A.; Sanyal, D. On the fractal nature of Penrose tiling. Curr. Sci. 2000, 79, $364-367$.

9. Yudin, V.; Karygina, Y. Fractal images of quasicrystals as an example of Penrose lattice. Crystallogr. Rep. 2001, 46, 922-926. [CrossRef]

10. Fang, F.; Irwin, K. An icosahedral quasicrystal as a golden modification of the icosagrid and its connection to the e8 lattice. arXiv 2015, arXiv:1511.07786.

11. Levine, D.; Steinhardt, P.J. Quasicrystals. I. definition and structure. Phys. Rev. B 1986, 34, 596. [CrossRef] [PubMed]

12. Douady, A.; Hubbard, J.H.; Lavaurs, P. Etude Dynamique des Polynômes Complexes; Université de Paris-Sud, Dép. de Mathématique: Orsay, France, 1984.

13. Devaney, R.L. The Fractal Geometry of the Mandelbrot Set. II. How to Count and How to Add. Available online: http:/ / math.bu.edu/DYSYS/FRACGEOM2/FRACGEOM2.html (accessed on 1 August 2019).

14. Strogatz, S.H. Nonlinear Dynamics and Chaos with Applications to Physics, Biology, Chemistry, and Engineering; Perseus Books: New York, NY, USA, 1994.

15. Bazley, T.S. Index to Geometric Chuck; Waterlow and Sons: London, UK, 1875.

(C) 2019 by the authors. Licensee MDPI, Basel, Switzerland. This article is an open access article distributed under the terms and conditions of the Creative Commons Attribution (CC BY) license (http:/ / creativecommons.org/licenses/by/4.0/). 